Portland State University

PDXScholar

$10-1-2012$

\title{
Experimental Validation of Phase Using Nomarski Microscopy With an Extended Fried Algorithm
}

\author{
Scott A. Prahl \\ Oregon Institute of Technology \\ Amanda Dayton \\ Oregon Health \& Science University \\ Kyle Juedes \\ Portland State University \\ Erik J. Sánchez \\ Portland State University \\ Rafael Paez López \\ Instituto Nacional de Astrofisica
}

See next page for additional authors

Follow this and additional works at: https://pdxscholar.library.pdx.edu/ece_fac

Part of the Electrical and Computer Engineering Commons Let us know how access to this document benefits you.

\section{Citation Details}

Prahl, S. A., Dayton, A., Juedes, K., Sanchez, E. J., Lopez, R. P., \& Duncan, D. D. (2012). Experimental validation of phase using Nomarski microscopy with an extended Fried algorithm. Journal of the Optical Society of America a-Optics Image Science and Vision, 29(10), 2104-2109.

This Article is brought to you for free and open access. It has been accepted for inclusion in Electrical and Computer Engineering Faculty Publications and Presentations by an authorized administrator of PDXScholar. Please contact us if we can make this document more accessible: pdxscholar@pdx.edu. 


\section{Authors}

Scott A. Prahl, Amanda Dayton, Kyle Juedes, Erik J. Sánchez, Rafael Paez López, and Donald D. Duncan 


\title{
Experimental validation of phase using Nomarski microscopy with an extended Fried algorithm
}

\author{
Scott A. Prahl, ${ }^{1,2, *}$ Amanda Dayton, ${ }^{2}$ Kyle Juedes, ${ }^{3}$ Erik J. Sánchez, ${ }^{3}$ \\ Rafael Páez López, ${ }^{4}$ and Donald D. Duncan ${ }^{3}$ \\ ${ }^{1}$ Oregon Insitute of Technology, 27500 SW Parkway Avenue, Wilsonville, Oregon 97070, USA \\ ${ }^{2}$ Oregon Health \& Science University, 3303 SW Bond Avenue, Portland, Oregon 97239, USA \\ ${ }^{3}$ Portland State University, 1900 SW 4th Avenue, Portland, Oregon 97201, USA \\ ${ }^{4}$ Instituto Nacional de Astrofisica, Óptica y Electrónica, Tonantzintla, Puebla 72840, Mexico \\ *Corresponding author: scott.prahl@oit.edu
}

Received May 4, 2012; revised August 24, 2012; accepted August 25, 2012;

posted August 27, 2012 (Doc. ID 167993); published September 12, 2012

\begin{abstract}
Reconstruction of an image (or shape or wavefront) from measurements of the derivatives of the image in two orthogonal directions is a common problem. We demonstrate how a particular reconstructor, commonly referred to as the Fried algorithm, can be used with megapixel derivative images to recover the original image. Large datasets are handled by breaking the derivative images into smaller tiles, applying the Fried algorithm and stitching the tiles back together. The performance of the algorithm is demonstrated using differential interference contrast microscopy on a known test object. (c) 2012 Optical Society of America
\end{abstract}

OCIS codes: $\quad 120.5050,170.3660,170.6935,180.3170$.

\section{INTRODUCTION}

Historically, Nomarski or differential interference contrast (DIC) microscopy has been used only to derive qualitative phase information. The goal of our efforts is to acquire quantitative measurements of phase using an unmodified DIC microscope. In this paper, we assume that the phase is changing sufficiently slowly that the phase gradient varies smoothly over several resolution elements. This avoids both undersampling the wavefront and minimizing any diffractive effects. These effects are especially problematic because traditional coherent imaging techniques cannot be used for correction because the DIC white-light illumination is partially coherent.

Various researchers have been pursuing quantitative phase by a variety of means. For example, Kou et al. [1] have used the transport of intensity (TI-DIC) concept [2]. This idea relies on acquisition of images at focus and on either side of focus, and exploits the relationship between axial and transverse intensity gradients. Subsequently, phase is recovered through Fourier inversion of the Laplacian with regularization. This approach was demonstrated on a sample displaying no absorption, and the sensitivity of the approach to such absorption is an issue remaining to be explored. Another approach due to Preza et al. [3] uses DIC images at several azimuthal orientations to reconstruct phase. This so-called "rotational diversity" approach, similarly to the TI-DIC approach, requires the specimen to be nonabsorbing. The issue of absorbing specimens is avoided using the so-called "spiral phase integration" technique [3], which makes use of Fourier transform integration (with regularization) of the phase slopes. In essence, this is a Wiener filtering operation that is subject to low spatial frequency phase errors [4]. Shribak and Inoué [5] recovered relative phase with a phase-stepping approach, making use of the cosine relationship between the phase gradient and shear axis directions, and quasi phase stepping using a precision rotation stage. In principle, this approach could determine the absolute phase (within a constant), but this remains to be demonstrated. Finally, Dana [6] performed a bias calibration and chose a bias setting about which the relationship between phase and intensity was approximately linear. Of course this approach is limited to small phase excursions.

We previously introduced a technique to acquire quantitative phase derivative information using a DIC microscope [7]. Such an approach was useful for characterizing the first- and second-order directional scatter statistics for thin biological specimens. Here, we are concerned with integration of orthogonal phase slopes into a phase surface. We make use of the

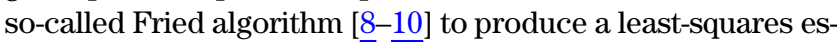
timate tiling of the phase surface that may extend to thousands of pixels in each direction.

The main drawback of the Fried reconstructor is that the size of the reconstruction kernel grows with the fourth power of the number of pixels on a side. Since this kernel must be inverted, large images cannot be handled directly. We avoid this problem by subdividing the image into smaller tiles having kernel sizes that permit rapid reconstruction on a desktop computer. Unfortunately, after reconstruction, the phase surface for each tile will have an unknown constant of integration. A second step, which we call "stitching," is used to determine the constants of all tiles at once by minimizing the differences along the edges. Once stitching is complete, the final phase surface is known to within a single overall arbitrary constant.

In the following discussion, we briefly review the Fried algorithm, discuss a strategy for stitching together the individually reconstructed image tiles, and demonstrate the performance of the algorithm on a known phase object of our construction. 


\section{BACKGROUND}

The Fried algorithm (Fig. 1) is based on expressing the gradients of the phase $\left(D x_{i, j}\right.$ and $\left.D y_{i, j}\right)$ at the pixel centers in terms of the phase $\phi_{i+1, j+1}$ specified at the vertices of these pixels,

$$
\begin{aligned}
& D x_{i, j}=\frac{\left(\phi_{i, j+1}+\phi_{i+1, j+1}\right)-\left(\phi_{i, j}+\phi_{i+1, j}\right)}{2}, \\
& D y_{i, j}=\frac{\left(\phi_{i+1, j}+\phi_{i+1, j+1}\right)-\left(\phi_{i, j}+\phi_{i, j+1}\right)}{2},
\end{aligned}
$$

where $i$ and $j$ are the pixel row and column. $D x_{i, j}$ and $D y_{i, j}$ have units of phase/pixel. The notation $\overline{\mathbf{X}}$ denotes a $n m \times 1$ vector that represents the $m \times n$ matrix $\mathbf{X}$ in column-major form,

$$
X_{i, j} \equiv \bar{X}_{m j-m+i}
$$

If the $\Phi$ consists of the phase elements $\phi_{i, j}$ and $\mathbf{D}_{x}$ and $\mathbf{D}_{y}$ contain the phase derivatives, then Eq. (1) can be rewritten

$$
\left[\begin{array}{l}
\mathbf{W}_{x} \\
\mathbf{W}_{y}
\end{array}\right] \bar{\Phi}=\left[\begin{array}{l}
\overline{\mathbf{D}}_{x} \\
\overline{\mathbf{D}}_{y}
\end{array}\right],
$$

where $\mathbf{W}_{x}$ and $\mathbf{W}_{y}$ are $m n \times(m+1)(n+1)$ sparse matrices with nonzero values of $\pm \frac{1}{2}$ specified by Eq. (1). If $\mathbf{W}^{-1}$ represents the pseudo-inverse of $\mathbf{W}$, then

$$
\bar{\Phi}=\mathbf{W}^{-1} \overline{\mathbf{D}},
$$

where $\mathbf{W}^{-1}$ has dimensions of $(m+1)(n+1) \times 2 m n$ and $\overline{\mathbf{D}}$ is a $2 m n \times 1$ vector of the (measured) gradients. The matrix $\Phi$ will be one row and one column larger than the derivative matrices, $\mathbf{D}_{x}$ and $\mathbf{D}_{y}$.

The dimensions $m$ and $n$ must be moderate to permit $\mathbf{W}^{-1}$ to be calculated. For example, if $m=n=32$, then $\mathbf{W}$ will have dimensions of $2048 \times 1089$ - a size that is readily inverted on current desktop computers. The number of elements in $\mathbf{W}$ grows as the fourth power of the linear dimension and $\mathbf{W}$ is prohibitively large for megapixel images. Since the number of operations required to invert a $N \times N$ matrix is $O\left(N^{2.4}\right)$,

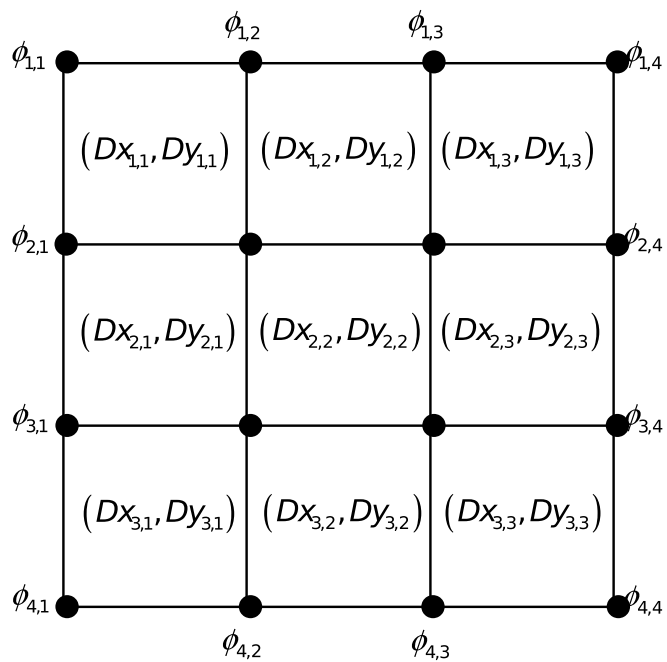

Fig. 1. Illustration of Fried geometry. The derivative values $\left(D x_{i, j}\right.$, $D y_{i, j}$ ) for each pixel are known and are related to the unknown values $\phi_{i, j}$ at each corner through Eq. (1). directly applying the Fried technique to a $1024 \times 1024$ image would take $10^{7}$ times as long as a $32 \times 32$ image.

To avoid the difficulty of reconstructing the entire phase matrix at once, we divide the large gradient matrices, $\mathbf{D}_{x}$ and $\mathbf{D}_{y}$, into a $k \times \ell$ grid of contiguous tiles, each of a convenient size, say $32 \times 32$. The phase matrix for each individual tile is then reconstructed. Unfortunately, an unknown constant of integration $c_{u, v}$ is associated with each tile and must be determined before the phase matrices for all the tiles may be recombined. The unknown constants form a $k \times \ell$ matrix $\mathrm{c}$ that is determined during the stitching process.

The reconstruction accuracy of the Fried technique at different spatial frequencies is shown in Fig. 3. To generate this figure, the $x$-and $y$-derivatives of the periodic phase grating

$$
\phi(x, y)=\sin [2 \pi(x+y) f]
$$

were found analytically. The derivatives were evaluated at pixel centers to generate phase gradient matrices $\mathbf{D}_{x}$ and $\mathbf{D}_{y}$. The reconstructed matrix $\Phi$ generated using the Fried method was compared with expected phases by calculating the standard deviation of the difference over a single $32 \times$ 32 tile. The Fried algorithm performs best when the phase gradients vary over several resolution elements. When the phase changes by $2 \pi$ over only three elements, the standard error is as large as the original signal; when the phase changes over six elements, the error drops to about $10 \%$. Even lower spatial frequencies resulted in even smaller reconstruction errors. Finally, the reconstruction errors for $8 \times 8$ and $16 \times 16$ tiles were essentially equivalent to those shown in Fig. 3 .

\section{TILE STITCHING STRATEGY}

The stitching process determines the unknown constants $c_{u, v}$ associated with the tile $\mathbf{M}_{u, v}$. The basis for our tile stitching algorithm is to use the overlap by one row and/or one column between the contiguous reconstructed tiles (Fig. 2). Specifically, we set the difference between $c_{u, v+1}$ and $c_{u, v}$ to the average difference between the pixels on the right edge of tile $\mathbf{M}_{u, v}$ and those on the left edge of tile $\mathbf{M}_{u, v+1}$ :

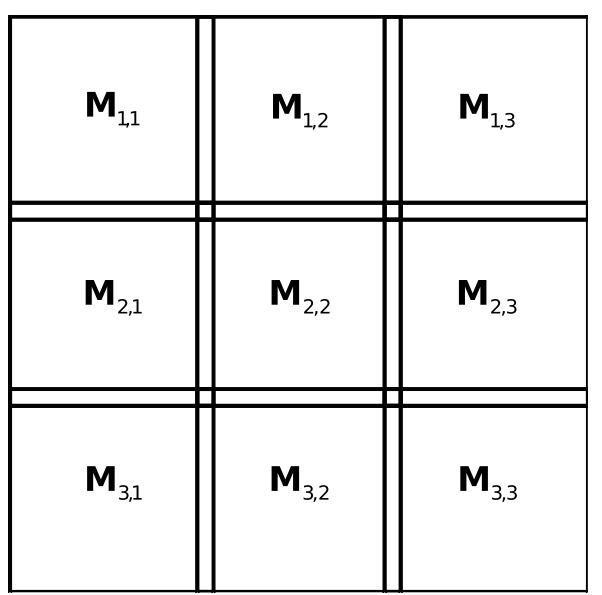

Fig. 2. Each tile $\mathbf{M}_{i j}$ is a $(m+1) \times(n+1)$ matrix of pixels and overlaps adjacent tiles. The unknown constant of integration $c_{i, j}$ for each tile is chosen to minimize the differences along the tile edges. 


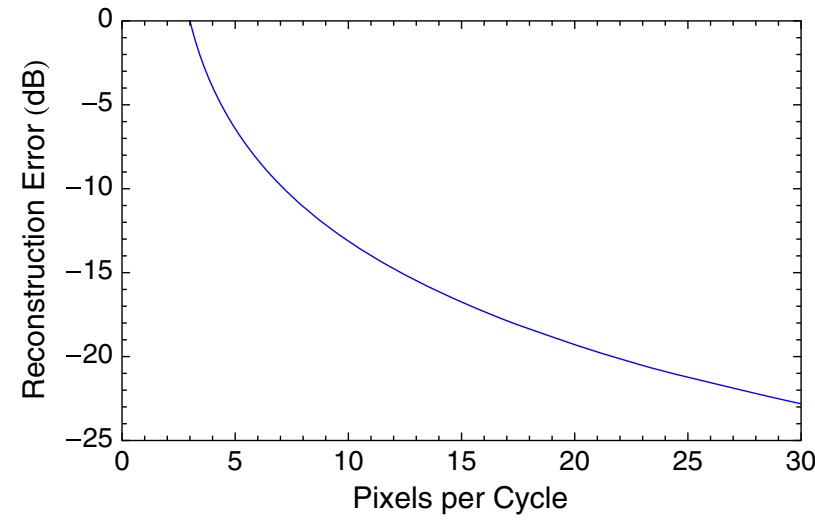

Fig. 3. (Color online) Intrinsic reconstruction error of the Fried algorithm for a periodic phase grating.

$$
c_{u, v+1}-c_{u, v}=\frac{1}{n} \sum_{i=1}^{m+1}\left(M_{u, v}^{i, n+1}-M_{u, v+1}^{i, 1}\right)=E x_{u, v},
$$

where $M_{u, v}^{i, j}$ designates the $(i, j)$ element in tile $\mathbf{M}_{u, v}$. Similarly, the difference between the top and bottom of adjacent vertical tiles is

$$
c_{u+1, v}-c_{u, v}=\frac{1}{n} \sum_{j=1}^{n+1}\left(M_{u, v}^{m+1, j}-M_{u+1, v}^{1, j}\right)=E y_{u, v} .
$$

This set of equations can be written as

$$
\left[\begin{array}{l}
\mathbf{V}_{x} \\
\mathbf{V}_{y}
\end{array}\right] \overline{\mathbf{c}}=\left[\begin{array}{c}
\overline{\mathbf{E}}_{x} \\
\overline{\mathbf{E}}_{y}
\end{array}\right]
$$

where the error matrices $\mathbf{E}_{x}$ and $\mathbf{E}_{y}$ have dimensions $k \times \ell$ and so $\overline{\mathbf{E}}$ has dimensions $2 k \ell \times 1$. Eq. (6) can be written as

$$
\mathbf{V} \overline{\mathbf{c}}=\overline{\mathbf{E}},
$$

and the $[(k-1) \ell+k(\ell-1)] \times k \ell$ sparse system matrix $\mathbf{V}$ has only \pm 1 as nonzero entries and is determined by Eqs. (4) and (ㄷ). For instance, the $3 \times 3$ subset of tiles in Fig. $\underline{2}$ is

$$
\mathbf{V}_{x}=\left[\begin{array}{ccccccccc}
-1 & 0 & 0 & +1 & 0 & 0 & 0 & 0 & 0 \\
0 & -1 & 0 & 0 & +1 & 0 & 0 & 0 & 0 \\
0 & 0 & -1 & 0 & 0 & +1 & 0 & 0 & 0 \\
0 & 0 & 0 & -1 & 0 & 0 & +1 & 0 & 0 \\
0 & 0 & 0 & 0 & -1 & 0 & 0 & +1 & 0 \\
0 & 0 & 0 & 0 & 0 & -1 & 0 & 0 & +1
\end{array}\right] .
$$

Again, in Eq. (7), we have more equations than unknowns (the tile constants of integration) and thus seek a least-squares solution using the pseudo-inverse $\mathbf{V}^{-1}$. Like the Fried algorithm, this system matrix is ill-conditioned and we resort to use of the singular value decomposition to compute the inverse. We next discuss performance of this reconstruction and tiling algorithm on a known phase object.

\section{MATERIALS AND METHODS}

\section{A. Test Object}

The test object was fabricated using thermal evaporation. Deposition was performed with a modified Edwards E-306 coating system, with a base pressure of $9 \times 10^{-7}$ Torr. Due to the thickness of coating required, a large shielded tantalum boat was used; the size increase required modification of the power to four times the normal current capability of the original system, from 100 to $400 \mathrm{~A}$. A razorblade attached to a Daedal, Inc. linear translator was utilized to make the steps. The blade, which was close to the sample substrate, was moved manually using a rotational feedthrough in the chamber. The glass substrate was a $170 \mu \mathrm{m}$ thick glass cover slip (Erie Scientific Gold Seal \#3307), and the steps were fabricated with magnesium fluoride powder (Johnson Matthey Electronics, 98\% magnesium fluoride \#46102) placed in a tantalum boat (R. D. Mathis). Typical coating times were approximately $60 \mathrm{~s}$ per layer, with a coating rate of $1.0 \mathrm{~nm} / \mathrm{s}$; process pressure was roughly $10^{-5}$ Torr

Once the steps were fabricated and determined not to be under high stress (as evinced by crazing), the thickness of the test object was scanned using an atomic force microscope (AFM, Park Scientific M5). The thickness accuracy was verified by contact-mode AFM using a calibration standard (Nanosurf BT00250). The phase of the test object (relative to air) was calculated using the measured thicknesses
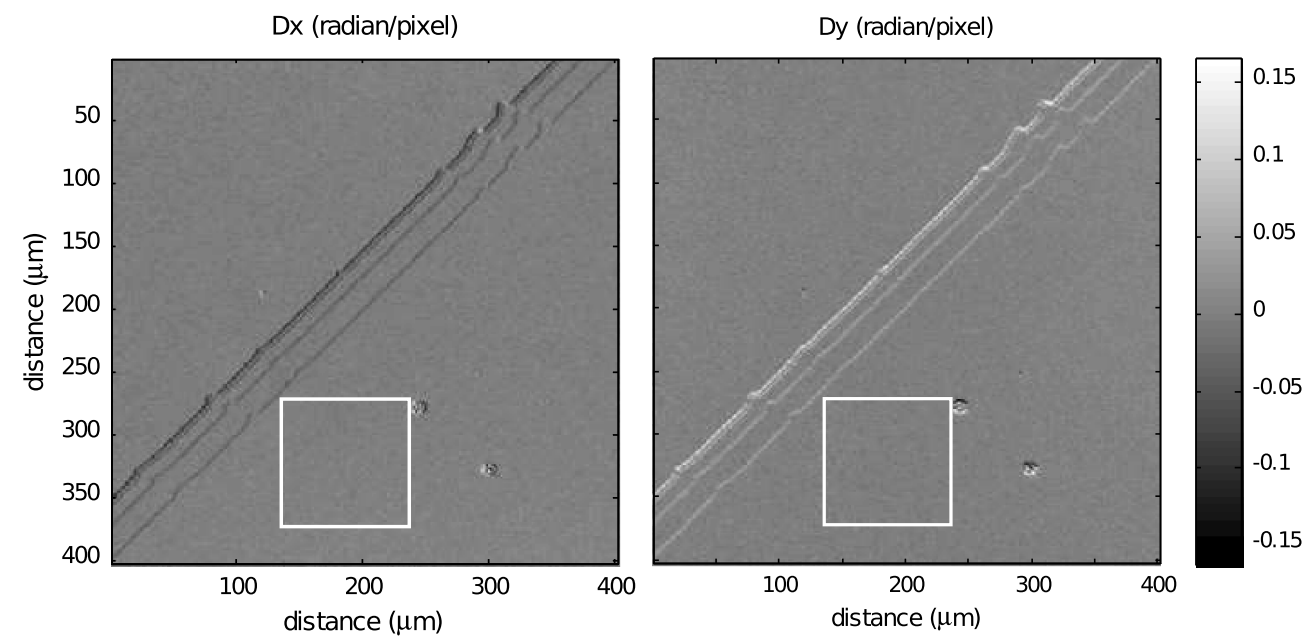

Fig. 4. Phase derivatives $\mathbf{D}_{x}$ and $\mathbf{D}_{y}$ obtained from DIC measurements of the test object. The white square shows the area on the bottom (uncoated) step that was averaged and subtracted to set the phase for this step to zero. 


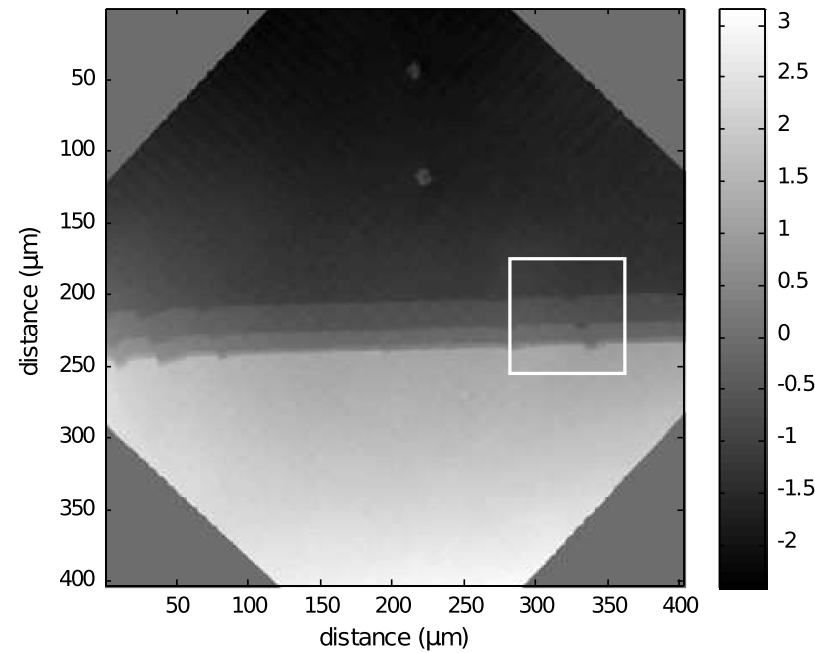

Fig. 5. Recovered phase in radians. The square indicates the region that corresponds to the area measured with the AFM.

$h(x, y)$ and the published [11] refractive index for magnesium fluoride, $n(0.405 \mu \mathrm{m})=1.3895$,

$$
\phi(x, y)=\frac{2 \pi h(x, y)}{\lambda}[n(\lambda)-1]
$$

\section{B. DIC Microscopy}

Each DIC image was acquired using a Zeiss Axio Imager with an Epiplan NeoFluar HD DIC 10× (0.3 NA) objective. The microscope was set up to provide Köhler illumination. The illuminating white light passed through a linear polarizer, a $405 \mathrm{~nm}$ bandpass filter, and a condenser Nomarski prism (DIC-I) before reaching the $\mathrm{MgF}_{2}$ test object. The bias/phase offset for each image was controlled using the set screw in the housing of the objective Nomarski prism. Images were captured with a color camera (Nikon Digital Sight, DS-Fi1). The exposure on the camera was manually set to $1 / 250 \mathrm{~s}$. All images were $2560 \times 1920$ pixels.

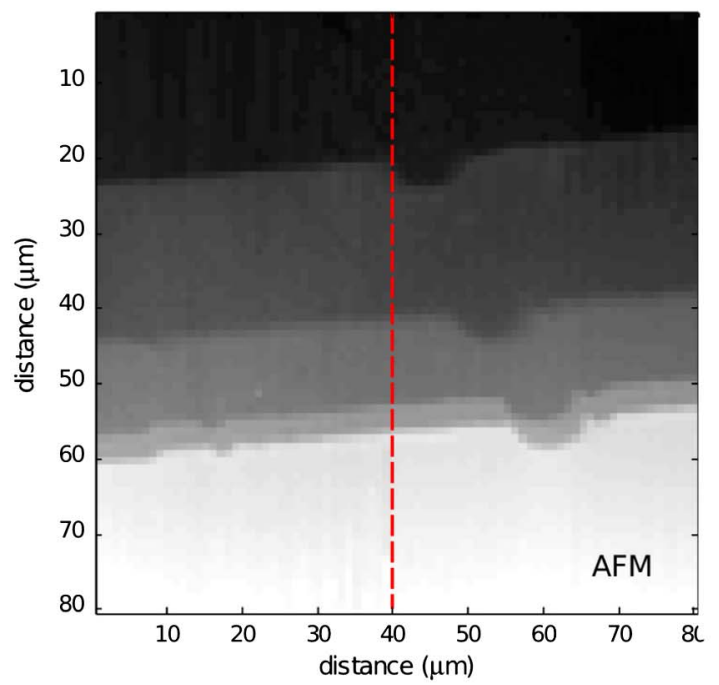

\section{Phase Gradient Images}

Gradient images in the two orthogonal directions were acquired using a DIC microscope using the methods described in Duncan et al. [7]:

1. acquire four DIC images with evenly spaced bias settings (one full turn of the bias screw on the objective Nomarski prism),

2. acquire four more images with the sample rotated so that the DIC shear is at $90^{\circ}$ with respect to the first group,

3. rotate the images so that the direction of shear is along the rows or columns of each image,

4. register the 0 and the $90^{\circ}$ images,

5. do Carré phase stepping calculations to extract the phase gradients in the $x$ and $y$ directions [7],

6. subtract a constant background phase gradient from the gradient images, and

7. scale the phase gradients by the shear and pixel size to obtain $\mathbf{D}_{x}$ and $\mathbf{D}_{y}$ with dimensions of radians/pixel [12],

The shear of the microscope at $\lambda=0.405 \mu \mathrm{m}$ was $2.4 \lambda \approx 0.97 \mu \mathrm{m}$. The pixel size was $2.95 \mu \mathrm{m}$. The starting phase derivative images are shown in Fig. 4 .

The gradient images obtained in step 6 above were also processed using spiral phase integration [4]. We did not include mirror reflecting to mitigate edge effects, nor did we window Arnison's $H(m, n)$ function to reduce high-frequency noise, because neither issue arose.

\section{RESULTS}

The Carré algorithm recovers the phase gradients to within an overall constant. The background constant for the phase gradients is determined by selecting a portion of the image (square in Fig. 4) that is known to have a constant valuein our case a portion of the lowest step not coated with $\mathrm{MgF}_{2}$.

The derivative images $\mathbf{D}_{x}$ and $\mathbf{D}_{y}$ were subdivided into $32 \times$ 32 tiles. The phase across each tile was acquired using Eq. (2). The unknown constants of integration were acquired using Eq. (7) and added to the elements of each tile. The phase tiles

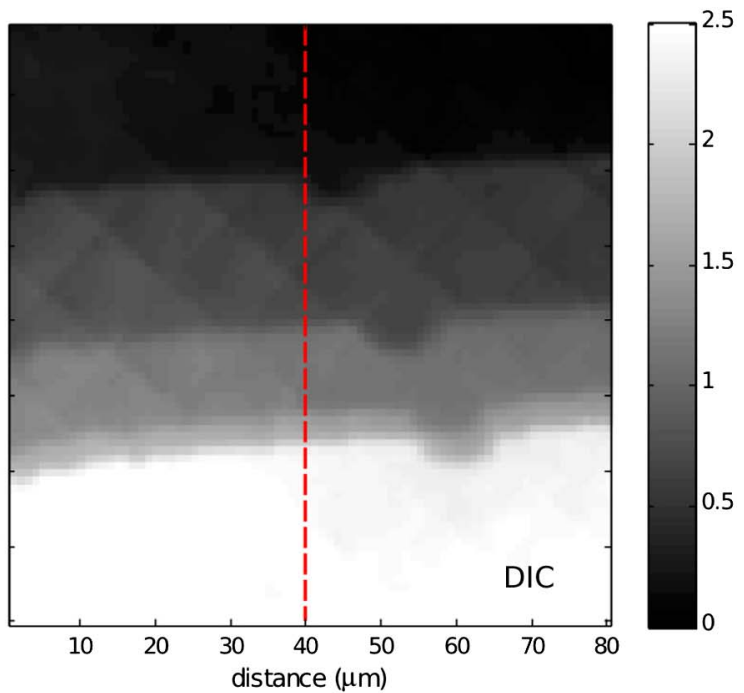

Fig. 6. (Color online) Phase estimates obtained using AFM (left) and DIC (right). The color bar on the right has units of radians. The vertical dashed line indicates the phases graphed in Fig. 7 . 


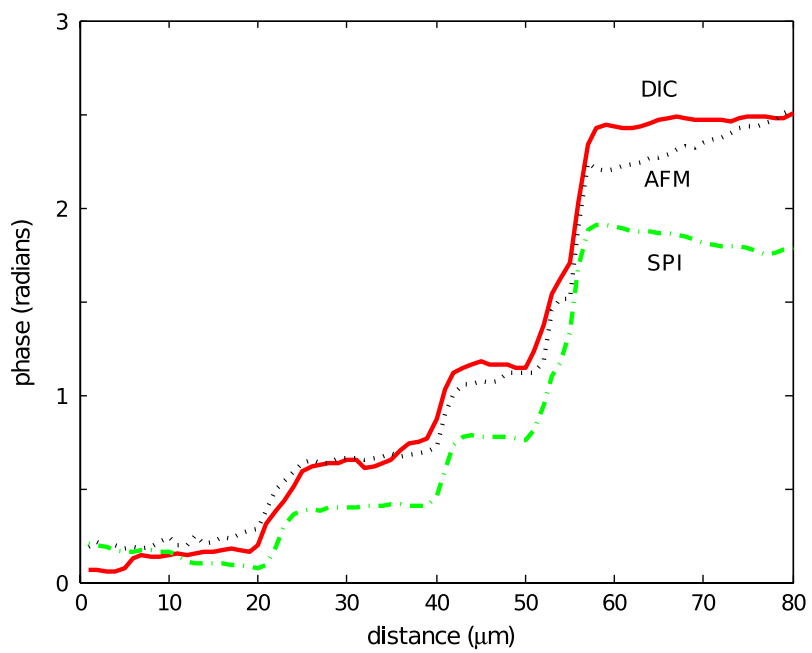

Fig. 7. (Color online) Comparison phases obtained with the DIC, AFM, and SPI (spiral phase integration) techniques along the dashed lines shown in Fig. $\underline{6}$.

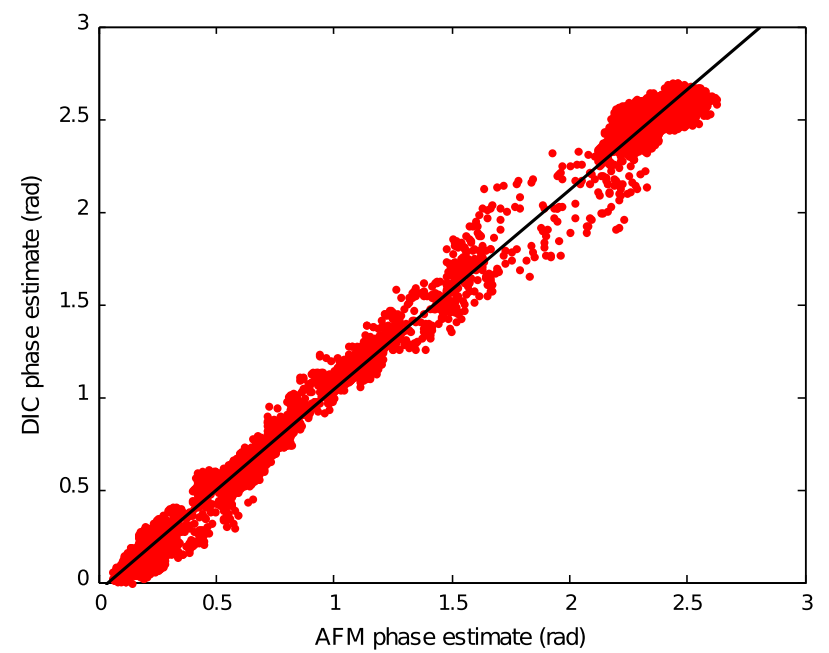

Fig. 8. (Color online) Relationship between the phases of each pixel determined using AFM (horizontal axis) and DIC (vertical axis) methods. The least-squares fit is $\phi_{\mathrm{DIC}}=-0.045+1.08 \phi_{\mathrm{AFM}}$.

were then recombined into a single large phase image (Fig. $\underline{5}$ ) using the tile stitching strategy described above.

The square in Fig. 5 shows the area of the test object scanned using the AFM. The AFM and the DIC phase estimates are shown in Fig. 6. The spiral phase integration result was visually indistinguishable from the AFM result and is not shown. Figure 7 is a comparison of the staircase phase profiles along the dashed lines in Fig. 6 . Also included in this figure are the spiral phase integration results along the same line of pixels. Finally, Fig. 8 shows the correlation of the AFM and DIC phase estimates for every pixel in Fig. $\underline{6}$.

\section{DISCUSSION AND CONCLUSIONS}

As seen in Figs. 7 and 8, the agreement between the DICderived phase estimate and that of the test object is very good; correlation between phase estimates (Fig. 8) exceeds 0.99. The $8 \%$ slope discrepancy can be attributed to uncertainty in the estimate of the background phase gradient. The spiral phase integration result is surprisingly good considering that this phase object has significant low frequency content (which is filtered out by algorithm).

We believe this is the first demonstration of the reconstruction of the quantitative phase of a tenuous object (analogous to a thin biological specimen) using DIC phase stepping with an unmodified microscope. Our particular approach has some drawbacks: the need to collect eight DIC images, image registration, manual rotation of the sample, and subtraction of the global phase gradient. Further, our stitching algorithm also has matrices that grow rapidly with the number of tiles used. On the other hand, there are a number of distinct advantages to our approach. The Fried matrices are sparse and only need to be inverted once, the Carré approach is expected to be insensitive to absorption effects, and real biological samples are not expected to exhibit background phase gradients.

One solution to the stitching algorithm described above would be a straightforward pyramid application of the algorithm. Another alternative that we have used is a sequential stitching process that proceeds in a raster or serpentine fashion. Such an algorithm performs nearly as well, and can be applied to arbitrarily large images. Many of the disadvantages of our approach can be obviated using active optical components (that are not available in conventional DIC microscopes). Finally, we have demonstrated an unambiguous experimental validation of the concept.

Errors in the reconstruction algorithm are composed of both intratile (reconstructor-based, Fig. 3) and intertile (stitching-based) components. The phase gradient between steps in the test image is too low (about 28 pixels /cycle at the steepest step) to have significant intra-tile errors. However, the intertile artifacts can be seen in the reconstruction of Fig. 5. Other reconstructors are possible, such as the Hudgin $[\underline{13}, \underline{14}]$ or Southwell [15]. For the Hudgin reconstructor, the intratile root-mean-square errors are lower than for the Fried reconstuctor. However, the reconstructed tiles (as in the Southwell case) have the same dimensions as the gradient tiles. Consequently, there is not an extra row and column that overlap adjacent tiles, and the errors arising from tile stitching are higher. For use of these alternative reconstructors, the strategy for developing the error vector, $\overline{\mathbf{E}}$, may have to be modified. 
Other effects remaining to be explored are those due to misregistration between the gradient images, noise, and the number of quantization levels. The effect of these details can be characterized through simulation using a series of sinusoidal fringe patterns and in terms of a global or local optical transfer function $[16,17]$. Results of these studies will be the subject of a future publication.

\section{ACKNOWLEDGMENTS}

RPL was supported through a grant from El Consejo Nacional de Ciencia y Tecnología, and SAP was partially supported by National Institutes of Health grant NIH-NIDCR-R21DE016758.

\section{REFERENCES}

1. S. S. Kou, L. Waller, G. Barbastathis, and C. J. R. Sheppard, "Transport-of-intensity approach to differential interference contrast (TI-DIC) microscopy for quantitative phase imaging," Opt. Lett. 35, 447-449 (2010).

2. M. R. Teague, "Deterministic phase retrieval: a Green's function solution,” J. Opt. Soc. Am. 73, 1434-1441 (1983).

3. C. Preza, S. V. King, and C. J. Cogswell, "Algorithms for extracting true phase from rotationally-diverse and phase-shifted DIC images," Proc. SPIE 6090, 60900E (2006).

4. M. R. Arnison, K. G. Larkin, C. J. R. Sheppard, N. I. Smith, and C. J. Cogswell, "Linear phase imaging using differential interference contrast microscopy," J. Microsc. 214, 7-12 (2004).

5. M. Shribak and S. Inoué, "Orientation-independent differential interference contrast microscopy," Appl. Opt. 45, 460-469 (2006).

6. K. J. Dana, "Three dimensional reconstruction of the tectorial membrane: an image processing method using Nomarski differential interference contrast microscopy," Master's thesis (Massachusetts Institute of Technology, 1992).

7. D. D. Duncan, D. G. Fischer, A. Dayton, and S. A. Prahl, "Quantitative Carré differential interference contrast microscopy to assess phase and amplitude," J. Opt. Soc. Am. A 28, 1297-1306 (2011).

8. D. L. Fried, "Least-square fitting a wave-front distortion estimate to an array of phase-difference measurements," J. Opt. Soc. Am. 67, 370-375 (1977).

9. D. L. Fried, "Branch point problem in adaptive optics," J. Opt. Soc. Am. A 15, 2759-2768 (1998).

10. D. L. Fried, "Adaptive optics wave function reconstruction and phase unwrapping when branch points are present," Opt. Commun. 200, 43-72 (2001).

11. W. J. Tropf, M. E. Thomas, and T. J. Harris, "Properties of crystals and glasses," in Handbook of Optics, Volume II: Devices, Measurements, \& Properties, 2nd ed. (McGraw-Hill, 1995), Chap. 33, pp. 33.3-33.101.

12. D. D. Duncan, D. G. Fischer, M. Daneshbod, and S. A. Prahl, "Differential interference contrast microscopy for the quantitative assessment of tissue organization," Proc. SPIE 7570, $75700 \mathrm{C}(2010)$.

13. R. H. Hudgin, "Wave-front reconstruction for compensated imaging," J. Opt. Soc. Am. 67, 375-378 (1977).

14. R. J. Noll, "Phase estimates from slope-type wavefront sensors," J. Opt. Soc. Am. 68, 139-140 (1978).

15. W. H. Southwell, "Wave-front estimation from wave-front slope measurements," J. Opt. Soc. Am. 70, 998-1006 (1980).

16. D. J. Cuccia, F. Bevilacqua, A. J. Durkin, and B. J. Tromberg, "Modulated imaging: quantitative analysis and tomography of turbid media in the spatial-frequency domain," Opt. Lett. 30, 1354-1356 (2005).

17. D. J. Cuccia, F. Bevilacqua, A. J. Durkin, F. R. Ayers, and B. J. Tromberg, "Quantitation and mapping of tissue optical properties using modulated imaging," J. Biomed. Opt. 14, 024012 (2009). 